Anaesthesist 2018 67:893-894

https://doi.org/10.1007/s00101-018-0517-3

(c) Springer Medizin Verlag GmbH, ein Teil von Springer Nature 2018

CrossMark
W. Schaffartzik

Berlin, Deutschland

\title{
Die neue Zusatz-Weiterbildung Klinische Akut- und Notfallmedizin
}

\author{
Meilen- oder Stolperstein in der \\ Verbesserung der Behandlungsqualität in \\ Notaufnahmen?
}

Bisher konnten sich Ärzte in der präklinischen Notfallmedizin weiterbilden und nach der Erfüllung der in der Weiterbildungsordnung genannten Voraussetzungen die Bezeichnung ZusatzWeiterbildung (ZWB) Notfallmedizin erwerben. Durch die Verabschiedung der (Muster-)Weiterbildungsordnung (MWBO) der Bundesärztekammer - so geschehen und publiziert am 16.11.2018 - steht den Ärzten mit der ZWB Klinische Akut- und Notfallmedizin nun erfreulicherweise eine weitere Weiterbildungsmöglichkeit für diesen Bereich zur Verfügung [1].

Nicht unerwähnt sollte bleiben, dass im Bereich der Ärztekammer Berlin bereits im Jahr 2014 die Einführung der ZWB Klinische Notfall- und Akutmedizin beschlossen und im darauffolgenden Jahr eingeführt wurde; offensichtlich erfolgreich, wie der Präsident der Ärztekammer Berlin auch aus heutiger Sicht kommentiert:

Die Zusatz-Weiterbildung Notfall- und Akutmedizin hilft entscheidend, die $\mathrm{Pa}$ tientenversorgung in den Notaufnahmen der Berliner Krankenhäuser höchstwertig $z u$ gewährleisten. Die Befugten verfügen über ein sehr weites Spektrum klinischer Erfahrung und Routine und sind auch für die Weiterbildung in anderen Gebieten, bei denen die Rotation in die Notaufnahme erfolgt, sehr beliebt [3].
Gries et al. beschreiben den langen Weg zur Aufnahme der ZWB Klinische Notfall- und Akutmedizin in die MWBO [2]. Die Länge des Weges mag auch darin begründet gewesen sein, dass am Anfang die Idee von der Einführung eines Facharztes für Notfallmedizin stand, der in einigen anderen europäischen Staaten bereits etabliert war und inhaltlich den präklinischen und klinischen Teil der Notfallmedizin abgedeckt hätte. Dieser Idee war jedoch von verschiedenen Fachgebieten widersprochen worden. Kontroverse Diskussionen schlossen sich an, an deren Ende dann aber auch bei uns eine zunehmende und fachgebietsübergreifende Akzeptanz der Sinnhaftigkeit einer Zusatzweiterbildung (und eben nicht eines Facharztes) im Bereich der klinischen Akut- und Notfallmedizin stand.

Entsprechend ist es sehr erfreulich, dass nun mit der Einführung der ZWB Klinische Akut- und Notfallmedizin eine interdisziplinäre Basis gefunden werden konnte und in die MWBO aufgenommen worden ist. Diese ZWB hat das große Potenzial, Weiterzubildenden die Kenntnisse, Kompetenzen und Fähigkeiten zu vermitteln, die Voraussetzung dafür sind, die Abläufe in den Notaufnahmen der Krankenhäuser stetig besser zu strukturieren, die Behandlungsqualität zu erhöhen und damit zur Verbesserung des Ergebnisses für die Patienten zu kommen. Ob damit auch die von verschiedenen Stellen als überlang kritisierten Verweildauern von Patienten in Notaufnahmen verkürzt werden können, hängt allerdings auch von anderen Faktoren ab; nicht zuletzt vom Patienten selbst: Die allzu oft geübte Praxis, bei einer akuten Erkrankung eher eine Notaufnahme als den Hausarzt aufzusuchen, muss beendet werden. Eine Hilfe hierbei könnte die weitere Verbreitung sog. Portalpraxen sein, die in Krankenhäusern in Zusammenarbeit mit der kassenärztlichen Vereinigung etabliert werden.

Die MWBO 2018 definiert die ZWB Klinische Akut- und Notfallmedizin wie folgt: „Die Zusatz-Weiterbildung Klinische Akut- und Notfallmedizin umfasst in Ergänzung zu einer Facharztkompetenz die Erstdiagnostik und Initialtherapie von Notfall- und Akutpatienten im Krankenhaus sowie die Indikationsstellung und Koordination der weiterführenden fachspezifischen Behandlung in interdisziplinärer Zusammenarbeit" [1]. Hierfür sind die Facharztanerkennung auf einem Gebiet der unmittelbaren $\mathrm{Pa}$ tientenversorgung und eine 6-monatige intensivmedizinische Weiterbildung erforderlich. Zusätzlich ist ein 80-StundenKurs in allgemeiner und spezieller Notfallbehandlung $\mathrm{zu}$ absolvieren. Dieser Kurs wird sicherlich Gemeinsamkeiten mit dem 80-Stunden-Kurs für die ZWB Notfallmedizin aufweisen. Wie Gries et al. ausführen, muss ein derartiger Kurs u.a. aber auch die strukturellen und organisatorischen Belange einer Notaufnahme vermitteln [2]. Außer- 
dem sind von den Weiterzubildenden 24 Monate Weiterbildungszeit in einer interdisziplinären Notfallaufnahme zu absolvieren.

Da es sich um eine im Sinne der Fachgebiete interdisziplinäre ZWB handelt, sind den Weiterzubildenden Kenntnissen in nahezu allen medizinischen Fachgebieten, wie z. B. im Bereich der Onkologie, der Immunologie, der Dermatologie und der Psychiatrie zu vermitteln.

Gries at al. vermuten, dass die Übernahme und Umsetzung der ZWB Klinische Akut- und Notfallmedizin durch die Landesärztekammern (LÄK) noch 2 Jahre in Anspruch nehmen könnten [2]. Die LÄK haben die Möglichkeit, den Inhalt der MWBO-ZWB zu modifizieren. $\mathrm{Zu}$ wünschen wäre, dass die Diskussionen um Modifikationen zwischen den LÄK koordiniert würden und zu möglichst einheitlichen Ergebnissen führten.

Die ZWB Klinische Notfall- und Akutmedizin wird dazu beitragen, die in den Notaufnahmen der Krankenhäuser tätigen Ärzte besser zu qualifizieren als bisher. Erstrebenswert wäre, dass diese ZWB nicht nur von leitenden Ärzten von Notaufnahmen erworben wird, sondern auch von den Ärzten, die in Notaufnahmen langfristig tätig sind. Ärzte klinischer Fachgebiete, aber auch wir Anästhesisten haben nun verstärkt die Möglichkeit, nicht nur punktuell zu Behandlungen einzelner Patienten in der Notaufnahme hinzugezogen zu werden, sondern durch eine langfristige Tätigkeit in der Notaufnahme unseren Beitrag zu der Verbesserung der Behandlung der Patienten in Notaufnahmen zu leisten.

$\mathrm{Ob}$ die neue Weiterbildungsoption ein Meilenstein wird, wird sich erst entscheiden, wenn die ZWB Klinische Akut- und Notfallmedizin auf breite Akzeptanz trifft, also sich ein erheblicher Anteil der angesprochenen Ärzte, auch Anästhesisten, um diese Zusatzqualifikation bemüht, und dies dazu beiträgt, die Behandlungsqualität zu verbessern.

\section{Korrespondenzadresse}

\section{Prof. Dr. med. W. Schaffartzik}

Klistostraße 13, 14165 Berlin, Deutschland

walter.schaffartzik@gmail.com
Interessenkonflikt. W. Schaffartzik gibt an, dass kein Interessenkonflikt besteht.

\section{Literatur}

1. Bundesärztekammer (2018) (Muster-)Weiterbildungsordnung.http://www.bundesaerztekammer. de/aerzte/aus-weiter-fortbildung/weiterbildung/ muster-weiterbildungsordnung/. Zugegriffen: 20.11.2018

2. Gries A, Seekamp A, Wrede C et al (2018) ZusatzWeiterbildung Klinische Akut- und Notfallmedizin in Deutschland - ein Konzept vor der bundesweiten Einführung! Anaesthesist. https://doi.org/10. 1007/s00101-018-0515-5

3. Jonitz G, Persönliche Mitteilung. 20. November 2018
Hier steht eine Anzeige. Springer 